\title{
Diálogos sobre a Construção dos Itinerários Terapêuticos por Pessoas que Vivenciam Hanseníase
}

\author{
Fábio Solon Tajra ${ }^{1}$, Guilherme de Sousa Lima², Eduardo Roesener \\ Vieira $^{2}$, Camila Maria Coelho de Moura ${ }^{2}$, Viriato Campelo ${ }^{3}$ \\ ${ }^{1}$ Departamento de Medicina Comunitária da Universidade Federal do Piauí, Centro de \\ Inteligência em Agravos Tropicais Emergentes e Negligenciados, Brasil | \\ fstajra@hotmail.com | https://orcid.org/0000-0001-7236-5541 \\ 2 Universidade Federal do Piauí, Brasil | guilhermedes|@outlook.com; \\ eroesenerv@gmail.com; camilacoelho0523@gmail.com | https://orcid.org/0000-0002-0986- \\ 6797; https://orcid.org/0000-0003-4750-150X; https://orcid.org/0000-0001-5872-2602 \\ ${ }^{3}$ Departamento de Parasitologia e Microbiologia da Universidade Federal do Piauí, Brasil | \\ viriato.campelo@bol.com | https://orcidid.org/0000-0001-7236-5541
}

Resumo: A hanseníase, agravo negligenciado no Brasil, tem constituído um problema de saúde pública. Existe uma tensão para que sejam elaboradas propostas de intervenção para prevenção, controle e minimização dos efeitos ocasionados. Os itinerários terapêuticos construídos pelas pessoas que vivenciam esse agravo contribuem para a identificação de boas práticas, bem como dos pontos a serem melhorados e aperfeiçoados. Objetivos: compreender os itinerários terapêuticos da população que vivenciam hanseníase no estado do Piauí, tendo em vista a necessidade de perceber as potencialidades e fragilidades da rede. Métodos: Trata-se de um estudo de delineamento qualitativo norteado pelo paradigma interpretativo. Realizamos entrevistas individuais a partir de roteiro semiestruturado com treze pessoas que vivenciavam hanseníase no estado do Piauí. O projeto foi aprovado pelo Comitê Ética em Pesquisa da Universidade Federal do Piauí (no 3.429.590). Resultados: as unidades de Atenção Primária à Saúde foram mencionadas como importante referência para o acolhimento dos casos e condução do cuidado em saúde. Contudo, apresentaram fragilidades relacionados ao diagnóstico e preparo dos profissionais diante das condutas apropriadas. Conclusões: importante levar em consideração os destaques mencionados neste estudo, à medida em que se propõe o cuidado à saúde de pessoas que vivenciam a hanseníase. Isso inclui a reflexão sobre a atuação dos equipamentos de saúde, bem como a organização e o funcionamento dos serviços em rede, a definição de sistemas de apoio e logística e a adoção de protocolos e fluxos. Desse modo, é possível verificar a efetividade desses equipamentos de saúde na atenção integral ao paciente e propor intervenções que visem a melhoria desse serviço.

Palavras-chave: Hanseníase; Acesso Universal aos Serviços de Saúde; Integralidade; Integralidade em Saúde; Pesquisa Qualitativa; Hermenêutica.

\section{Dialogues on the Construction of Therapeutic Itineraries by People Who Experience Leprosy}

Abstract: Leprosy, neglected disease in Brazil, has been a public health problem. There is a tension for the elaboration of intervention proposals for prevention, control and minimization of the effects caused. The therapeutic itineraries built by the people who experience this condition contribute to the identification of good practices, as well as the points to be improved and perfected. Objetives: to understand the therapeutic itineraries of the population that experiences leprosy in the state of Piauí, in view of the need to understand the potential and weaknesses of the network. Methods: This is a qualitative design study guided by the interpretive paradigm. We conducted individual interviews based on a semi-structured script with thirteen people who were experiencing leprosy in the state of Piauí. The project was approved by the Research Ethics Committee of the Federal University of Piauí (number 3,429,590). Results: the Primary Health Care units were mentioned as an important reference for welcoming cases and conducting health care. However, they presented weaknesses related to the diagnosis and preparation of professionals in the face of appropriate conduct. Conclusions: it is important to take into account the highlights mentioned in this study, as it proposes health care for people who experience leprosy. This includes reflection on the performance of health equipment, as well as the organization and functioning of network services, the definition of support and logistics systems and the adoption of protocols and flows. In this way, it is possible to verify the effectiveness of these health equipment in comprehensive patient care and to propose interventions aimed at improving this service.

Keywords: Leprosy; Universal Access to Health Care Services; Integrality in Health Qualitative Research; Hermeneutics. 


\section{Introdução}

A hanseníase, agravo negligenciado no Brasil, tem constituído um problema de saúde pública. Sua ocorrência tem sido desproporcional em populações socioeconomicamente desfavorecidas e marginalizadas (Monteiro et al., 2017). Existe uma tensão para que sejam elaboradas propostas de intervenção para a sua prevenção, controle e minimização dos efeitos ocasionados. Contudo, são insuficientes e provocam pouco impacto na saúde coletiva (WHO, 2014).

A hanseníase constitui um agravo de transmissão direta que se relaciona com uma série de determinantes e condicionantes como, por exemplo, contato com o Mycobacterium leprae ou bacilo de Hansen associados à baixa renda, escassez alimentar, indisponibilidade de água tratada e coleta de lixo, dificuldade de acesso a programas de transferência de renda e aos serviços sociais de saúde (Monteiro et al, 2017; Silvestre \& Lima, 2016).

No Brasil, um dos países endêmicos, foi registrado, em 2020, uma taxa de detecção de 6,51/100.000 habitantes, além de uma taxa de detecção de incapacidade de grau 2 (IG2) de 5,23 por milhão de habitantes. Houve uma considerável queda da taxa de detecção em relação ao ano de 2019 (11,23 por 100.000 habitantes). Contudo, essa situação constitul um reflexo das barreiras impostas pela pandemia de COVID-19 (Coronavírus Disease) na detecção desses casos (Silva, 2021).

A Organização Mundial da Saúde (OMS) estabeleceu uma meta global de redução da detecção de IG2 para menos do que 1 por milhão de habitantes, o qual não foi atingido pelo Brasil até a presente data (World Health Organization, 2016). Aqui, faz-se necessário reunir esforços e aglutinar recursos de forma mais enérgica, tendo em vista a priorização do cuidado à saúde de pessoas que vivenciam a hanseníase.

Vale ressaltar que a hanseníase pode ocasionar efeitos diversos que exigem abordagem multiprofissional e práticas de saúde interdisciplinares. Isso tenciona para práticas plurais, aliada a articulação e integração dos serviços em redes. Aqui, as unidades de atenção primária à saúde (APS), conhecidas no país como Unidades Básicas de Saúde, tem papel decisivo na promoção, proteção e recuperação de saúde diante desse tipo de agravo. É porta de entrada e coordenadora do cuidado no sistema de saúde brasileiro. Contudo, são anunciados alguns problemas diante da perspectiva de cuidado em saúde (Sousa et al., 2017).

A partir disso, problematizamos: como se dão os itinerários terapêuticos de pessoas que vivenciam hanseníase? Quais equipamentos de saúde são acessados? Assim, o objetivo deste estudo é compreender os itinerários terapêuticos da população que vivencia hanseníase no estado do Piauí, tendo em vista a necessidade de perceber as potencialidades e fragilidades da rede.

\section{Metodologia}

Trata-se de um estudo de delineamento qualitativo norteado pelo paradigma interpretativo. Optamos por esse tipo de estudo por privilegiar os sentidos e os afetos dos movimentos de uma comunidade (Bosi; Mercado \& Martínez, 2004). No que diz respeito ao paradigma interpretativo, entendemos que as representações observadas até aquele momento foram construídas historicamente e são produto de um processo de interação entre os sujeitos. Nesse caso, a ênfase é dirigida ao processo e inclui a compreensão sobre as percepções e o significado que os fenômenos têm para cada um deles.

Vale mencionar que a escolha desse tipo de estudo esteve relacionada com a pergunta disparadora do estudo, aliada ao objetivo que pretendíamos responder e ao olhar curioso construído pelos pesquisadores. Essa tríade parece sustentar a escolha do método e técnica a ser utilizada (Bosi, Mercado \& Martínez, 2004). 
Para garantir a transparência e completude desta produção, seguimos a proposta do Standards for Reporting Qualitative Research (SRQR) que inclui itens referentes ao título, resumo, formulação do problema e pergunta de pesquisa, delineamento e métodos de coleta e análise de dados, resultados, interpretação, discussão e integração, bem como outras informações (Patias \& Hohendorff, 2019). Assim, partimos para a descrição de todos os aspectos metodológicos e justificativa de nossas escolhas. Vale mencionar que a adoção desses critérios não esteve restrita à fase de produção textual. Utilizamos esse referencial, também, para as fases de planejamento e programação da pesquisa.

Realizamos entrevistas individuais a partir de roteiro semiestruturado com treze pessoas que vivenciavam hanseníase no estado do Piauí. Os participantes foram recrutados em dois hospitais de referência de nível terciário para esse agravo no estado do Piauí durante a fase de acolhimento das consultas agendas ou internação. O período de recrutamento dos participantes se deu ainda entre agosto de 2019 e fevereiro de 2020, anteriormente ao registro dos primeiros casos de COVID-19 no Piauí.

No que diz respeito aos critérios de inclusão dos sujeitos, foram considerados os usuários que acessaram os serviços desses hospitais de referência do estado do Piauí para os agravos tropicais emergentes ou negligenciados e ter mais de 18 anos. Foram excluídos adolescentes, pessoas com transtornos psiquiátricos, previamente diagnosticados na tentativa de reduzir qualquer tipo de risco relacionado à pesquisa.

Elaboramos um roteiro com perguntas abertas que tratava dos itinerários terapêuticos construídos por cada um deles. Dentre os questionamentos elaborados, podemos citar: Como foi a sua experiência no acesso aos serviços de saúde? Como chegou até esse serviço de saúde? Qual foi o seu itinerário?

O recrutamento dos participantes se deu por conveniência até a saturação teórica (Fontanella et al., 2011). Isso significa dizer que o ponto de saturação da amostra esteve dependente indiretamente do referencial teórico usado pelos pesquisadores e do recorte do objeto e diretamente dos objetivos definidos para a pesquisa, do nível de profundidade a ser explorado (dependente do referencial teórico) e da homogeneidade da população estudada.

Os dados foram produzidos após imersão prévia dos pesquisadores nesses espaços e acompanhamento dos serviços.

Houve estudo piloto em que os pesquisadores passaram por experimentação do roteiro e desenvolvimento da técnica de entrevista. Um docente e dois discentes do curso de Medicina da Universidade Federal do Piauí conduziram a coleta de dados após fundamentação teórico-conceitual e metodológica oportunizada por meio de encontros semanais durante o primeiro semestre de 2019. Nesses encontros, foi possível identificar as principais necessidades e demandas dos pesquisadores sobre esse tipo de pesquisa, estabelecer um diálogo permanente para o seu desenvolvimento e planejar a pesquisa de campo propriamente dita de forma participativa.

As entrevistas tiveram uma média de trinta minutos de duração, foram gravadas em áudio, transcritas e analisadas em blocos de três. Acreditamos que a análise em blocos possibilita a identificação de boas práticas na condução das entrevistas, assim como os pontos a serem melhorados e aperfeiçoados. Auxilia, ainda, no direcionamento do trabalho e na abordagem dos participantes, tendo em vista a necessidade de responder à pergunta do estudo. A partir disso, é possível também perceber outras temáticas e desdobramentos que merecem destaque em outros estudos.

Os dados foram arquivados em um drive para garantir o acesso a todos os pesquisadores. As transcrições foram compartilhadas com os participantes para comentários e correções.

No que diz respeito aos procedimentos éticos, enquanto projeto de pesquisa, a investigação cumpriu com a Resolução 466/12 do Conselho Nacional de Saúde do Brasil para a obtenção do consentimento informado dos participantes e garantia da preservação da confidencialidade dos dados. O projeto foi encaminhado ao Comitê Ética em Pesquisa da Universidade Federal do Piauí, tendo seu parecer aprovado com o número 3.429.590. 
Os dados foram manipulados e armazenados com vistas a garantir sua segurança e o anonimato dos participantes. Vale ressaltar que não existia relacionamento prévio à pesquisa entre pesquisador/a e pesquisado/a. Aos participantes, foi garantido os processos éticos relevantes na coleta de dados e seu consentimento.

Dentre os procedimentos de análise de dados, adotamos o referencial teórico da hermenêutica de Hans-Georg Gadamer (2015) associada às contribuições de Paul Ricoeur (1976). Aqui, a análise e interpretação das narrativas se deram em três fases: leitura inicial do texto, leitura crítica e apropriação. A leitura inicial do texto teve como objetivo compreendê-lo de maneira superficial, por meio da percepção dos primeiros significados. Foi realizada várias vezes e sem julgamentos, de modo que o pesquisador apreendeu os significados e pode organizá-los. Logo após, foi realizada a leitura crítica com a finalidade de interpretar e compreender os prováveis significados imbuídos no texto. Por fim, a apropriação foi feita com a compreensão e assimilação da mensagem desvelada (Terra et al., 2009).

Dois discentes realizaram as transcrições das entrevistas e a codificação dos dados foi realizada pelo docente orientador. Houve conferência dos dados transcritos com os coletados, tendo em vista a garantia da integridade dos dados. Foi realizada a transcrição total e analisada na íntegra, com o objetivo de identificar as unidades de análise (unidades de significado). O paradigma interpretativo orientou a análise, uma vez que os pesquisadores estudaram as coisas em seus cenários naturais, tentando entender os fenômenos em termos dos significados que as pessoas a eles conferem (Denzin \& Lincoln, 2006).

As unidades foram geradas indutivamente sem a utilização de softwares. Os resultados foram discutidos com os coletadores de dados, docente orientador e outros dois integrantes, um discente e um docente com expertise no assunto. Para garantir a credibilidade e fidedignidade da análise de dados, utilizamos a checagem por membros (Patias \& Hohendorff, 2019).

Para garantir a confidencialidade dos participantes da pesquisa, utilizamos, para a sua identificação, uma característica marcante de sua narrativa (Tabela 1).

Tabela 1. Perfil dos participantes do estudo, características relacionadas, idade e local de residência.

\begin{tabular}{cccc}
\hline Participante & Característica & Idade & $\begin{array}{c}\text { Local de } \\
\text { residência }\end{array}$ \\
\hline 1 & O trabalhador & *sem informação & Zona urbana \\
2 & O paizão & *sem informação & Zona rural \\
3 & O esperançoso & *sem informação & Zona rural \\
4 & O imparável & 55 anos & Zona rural \\
5 & A precavida & 25 anos & Zona urbana \\
6 & O otimista & 36 anos & Zona rural \\
7 & O alegre & 62 anos & Zona rural \\
8 & O consciente & 44 anos & Zona urbana \\
9 & O paciente & 36 anos & *sem informação \\
10 & O honesto & 39 anos & Zona rural \\
11 & O dedicado & *sem informação & *sem informação \\
12 & O persistente & 45 anos & Zona urbana \\
13 & O sincero & 32 anos & Zona urbana \\
\hline
\end{tabular}


É importante acrescentar que, para a caracterização dos itinerários e identificação dos estabelecimentos de saúde mencionados pelos participantes, optamos por apresentar os dados a partir de uma figura com ênfase na sua notificação. Utilizamos o software Microsoft Exce際 para construção do modelo de gráfico de polaridade apresentado.

\section{Apresentação e discussão dos resultados}

A experiência das pessoas é indispensável para a produção dos discursos científicos diante da busca pelo significado das ações nas relações entre interpretante e interpretado (Geanellos, 2000). Essa experiência é a base da abordagem hermenêutica (Caprara, 2003). Aqui, buscamos o contato com as pessoas que vivenciavam hanseníase para compreender os caminhos percorridos, bem como as suas emoções, sentimentos e reações.

Diversos itinerários foram descritos pelas pessoas entrevistadas. Foi possível, assim, identificar os tipos de equipamentos acessados e a frequência em que foram citados (Figura 1).

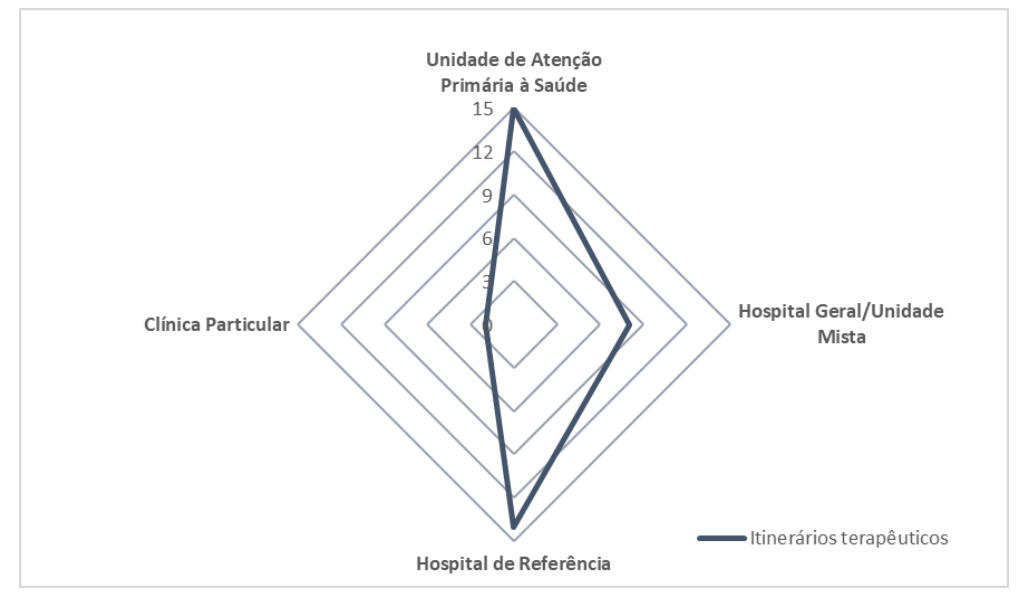

Fig. 1. Itinerários terapêuticos construídos por pessoas que vivenciam hanseníase.

O primeiro equipamento identificado foi a Unidade Básica de Saúde (UBS), responsável pela APS no sistema de saúde brasileiro. Starfield (2002) ressalta que a atenção no primeiro contato é um dos atributos essenciais da APS. É para este equipamento que, em geral, os participantes mencionaram se dirigir, quando perceberam sinais e sintomas sugestivos desse agravo:

[...] eu vim com um problema na perna [...] começou um inchaço e muita dormência [...] eu fui ao posto do bairro e a médica de lá analisou [...] ela ficou com a suspeita, porque eu tenho sempre uma manchinha ao redor [...] fez todos os testes que têm que fazer [...] tem o da hanseníase e tudo (A precavida).

[...] comecei com essas manchas no meu corpo [...] já tem seis anos [...] começou a tomar parte do corpo, nas pernas, no rosto [...] dormência [...] com a insistência da família eu fui pesquisar para ver o que era que estava se passando [...] eu fiz uma queimadura aqui [...] que eu não estava sentindo essa área do braço [...] peguei um ferro quente e trisquei [...] foi fundo, mas não senti a pele [...] eu disse: vou fazer o tratamento, porque isso aqui não é [...] normal [...] eu tive que ir no postinho perto de casa (O trabalhador).

Os profissionais da APS têm um papel importante diante do cuidado à saúde de pessoas que vivenciam hanseníase. Ações como a territorializacão, por exemplo, são fundamentais para o reconhecimento das necessidades e demandas da população. 
Uma vez reconhecidos casos de hanseníase no território, é possível elaborar um plano de ação específico e apropriado.

Aliado a isso, é preciso investir na promoção da saúde com ênfase em estratégias de educação, a fim de empoderar a população para reconhecer sinais e sintomas de patologias com incidência no território de atuação deste equipamento. Carnut (2017) comenta que a adoção de estratégias apropriadas às demandas da população deriva da relação da população com o território.

É importante que a pessoa reconheça os sinais e sintomas desse agravo pois, ao já apresentar perda de sensibilidade no momento do diagnóstico, a chance de desenvolver comprometimento da função nervosa e mão em garra aumenta em 65\% (Croft et al., 2000).

Outros equipamentos identificados foram o Hospital Geral e a Unidade Mista de Saúde, responsáveis por atendimentos de urgência/emergência e em especialidades básicas: [...] fui para o hospital no meu município e foi feito o exame lá mesmo [...] eu fiz e levei para a médica do postinho [...] lá, já começou o tratamento (O persistente).

Os serviços de urgência e emergência constituem pontos de atenção à saúde importantes dentro da rede e constituem porta de entrada. Nesse estudo, os participantes apontaram itinerários construídos a partir desses serviços. Aqui, houve articulação com outros setores com a apropriação dos profissionais de saúde da APS.

Em alguns relatos, foram percebidas fragilidades no diagnóstico e condução dos casos na APS e os profissionais buscaram referência para outros serviços especializados: [...] passei cinco meses com o problema sem tomar o remédio certo [...] depois, fui para o hospital [...] o doutor falou que tinha que ir para Teresina [...] lá tem um médico especialista nesse problema (O esperançoso).

A ocorrência do diagnóstico apenas pelo especialista, sendo por vezes precedida por diagnósticos equivocados na atenção primária, revela lacunas no atendimento integral ao paciente com hanseníase, além de retardar o diagnóstico do paciente e de aumentar as chances de desenvolver graves complicações. Para Alencar e Ramos (2012), existem lacunas na descentralização do atendimento integral ao portador da hanseníase, além de dificuldades com o acompanhamento durante e após a poliquimioterapia.

O terceiro equipamento de saúde identificado foi o Hospital de Referência. $\mathrm{O}$ atendimento dentro deste equipamento foi marcado por agilidade e satisfação do paciente: [...] (o médico) me encaminhou para cá [...] foi um anjo na minha vida [...] aqui (Hospital Universitário), a médica falou que estava com Hanseníase [...] no mesmo dia, fui diagnosticada e me passou o tratamento (O paizão); [...] eu vim em 2006 [...] fiz o exame aqui num dia e no outro dia já recebi o resultado [...] a doutora me falou: pode usar o remédio com fé que você vai ficar bom, mas só que você vai ficar com sequela $(\mathrm{O}$ esperançoso).

Vale mencionar que, nesses casos, as pessoas não obtiveram o diagnóstico na APS e precisaram ser encaminhadas para uma unidade hospitalar para fechar o diagnóstico junto a um especialista. Aqui, tivemos dúvidas quanto ao conhecimento profissional sobre o agravo e aos recursos existentes no município de origem para a elaboração do diagnóstico.

Apenas $9,1 \%$ dos municípios brasileiros contam com a assistência do especialista (Schmitt \& Miot, 2014). Essa realidade denota um empecilho para o acesso da população ao especialista, visto que a hanseníase é uma doença reconhecidamente associada a condições econômicas, sociais e ambientais desfavoráveis, mais marcantes em locais distantes dos grandes centros urbanos.

Um último equipamento de saúde identificado foram os consultórios e clínicas particulares, sempre associado com alguma lacuna do sistema de saúde, seja ela falta de exames ou do especialista: [...] fui fazer consulta lá na cidade, em Bom Jesus, e pediram um exame [...] eu fiz exame particular (O esperançoso). 
Todos esses itinerários foram atravessados por emoções, sentimentos e reações. Algumas narrativas foram atravessadas por desesperança, preocupação, medo, dúvida, mágoa, sensação de exclusão, sensação de impotência, desamparo, revolta, indignação, angústia e humilhação. Foram gerados a partir do desconhecimento do diagnóstico, da dificuldade de acesso aos serviços de saúde, da demora e da peregrinação. De fato, isso interfere diretamente na experiência de cuidado em saúde: "pensei até em desistir no começo, porque eles demoraram tanto" (O trabalhador).

Quando o acesso se materializou e a relação com o profissional de saúde esteve atravessada pela sensação de acolhimento e amparo, outras emoções e sentimentos foram percebidas: alívio, sensação de tranquilidade, satisfação e contentamento: "uma tranquilidade enorme [...] a doutora me diagnosticou e falou que não é uma doença que, assim, que passa [...] no meu caso [...] não é assim, muito agressivo e não é muito contagiosa, isso me deixa muito aliviado" (O paizão).

Para a perspectiva hermenêutica, as emoções, sentimentos e reações descritas por cada uma dessas pessoas revelam a procura de um novo significado de vida em contraposição aos aspectos que caracterizam a abordagem biomédica. Trata-se de um problema existencial, não somente de uma questão biologicista. A perspectiva de cura não pode ser a única a ser levada em consideração. Estamos tratando do reestabelecimento do bemestar e da qualidade de vida de cada uma dessas pessoas. A liberdade, por exemplo, é um componente importante a ser levado em consideração. Quando o acesso aos serviços de saúde é limitado ou a pessoa não contribui com a construção do seu plano terapêutico, isso é colocado em questão (Gadamer, 2006).

Na concepção gadameriana, o diálogo é um aspecto que deve ser levado em consideração quando se pretende buscar respostas para as necessidades e demandas de saúde de uma pessoa. Isso deve pautar a relação com o profissional de saúde. Gadamer propõe a experiência de uma medicina "humanista" que extrapola a técnica em si e analisa o ser humano na sua totalidade, o seu "ser no mundo" (Caprara, 2003; Gadamer, 2006).

A verificação dos itinerários terapêuticos é uma importante aliada na avaliação em saúde na perspectiva dos princípios da integralidade e da resolutividade em saúde (Vilela et al., 2019). A possibilidade de transformação das práticas de saúde depende dos encontros oportunizados em cada um dos itinerários terapêuticos. Assim, é indispensável apropriarse das narrativas das pessoas que vivenciaram esse agravo e construir novas formas de promoção do bem-estar, minimizando os efeitos gerados pela doença e sofrimento.

\section{Conclusões}

Compreendemos que os itinerários terapêuticos de pessoas que vivenciavam hanseníase estavam atravessados por dificuldades de acesso, peregrinação, fragilidades quanto ao diagnóstico e quanto à elaboração de um plano de cuidados em saúde. Associamos isso à falta de protocolos e linhas de cuidado adotadas no estado do Piauí e à frágil formação e desenvolvimento profissional.

É importante levar em consideração os destaques mencionados neste estudo, à medida em que se propõe o cuidado à saúde de pessoas que vivenciavam hanseníase. Isso inclul a reflexão sobre a atuação dos profissionais de saúde e construção de vínculos, bem como a disponibilidade e acesso aos equipamentos de saúde, a organização e o funcionamento dos serviços em rede, a definição de sistemas de apoio e logística e a adoção de protocolos e fluxos. Desse modo, foi possível verificar efeitos na integralidade em saúde.

Vale mencionar que esse trabalho pode contribuir com a discussão sobre os itinerários terapêuticos de pessoas que vivenciavam hanseníase por meio da abordagem hermenêutica. A perspectiva biomédica, contrária ao entendimento proposto por Gadamer, foi elucidada a partir da experiência de fragmentação do cuidado e da ênfase à cura. Foram desconsiderados aspectos importantes para a promoção do bem-estar de cada uma dessas pessoas. 
A abordagem hermenêutica nos permitiu explorar as experiências de pessoas que vivenciavam hanseníase e compreender como cada uma delas lidou com as barreiras de acesso aos serviços de saúde e com a peregrinação em busca do cuidado. Possibilitou, ainda, compreender as reações, sentimentos e emoções geradas pelas pessoas na construção da sua jornada.

\section{Referências}

Alencar, C. H. M., Ramos Júnior, A. N., Sena Neto, S. A., Murto, C., Alencar, M. J. F., Barbosa, J. C., \& Heukelbach, J. (2012). Diagnóstico da hanseníase fora do município de residência: uma abordagem espacial, 2001 a 2009. Cadernos de Saúde Pública, 28(9), 16851698. https://doi.org/10.1590/S0102-311X2012000900008

Bosi, M. L. M, \& Mercado, F.J. organizadores (2004). Pesquisa Qualitativa de Serviços de Saúde. Vozes.

Caprara, A. (2003). Uma abordagem hermenêutica da relação saúde-doença. Cad. Saúde Pública, Rio de Janeiro, 19(4):923-931.

Carnut, L. (2017). Cuidado, integralidade e atenção primária: articulação essencial para refletir sobre o setor saúde no Brasil. Saúde debate, 41(9), 1177-1186. https://doi.org/10.1590/0103 1104201711515

Croft, R. P., Nicholls, P. G., Steyerberg, E. W., Richardus, J. H., Cairns, W., \& Smith, S. (2000). A clinical prediction rule for nerve-function impairment in leprosy patients. Lancet (London, England), 355(9215), 1603-1606. https://doi.org/10.1016/s0140-6736(00)02216-9

Denzin, N. K., \& Lincoln, Y. S (2006). Introdução: a disciplina e a prática da pesquisa qualitativa. Em N. K. Denzin (Ed.). O planejamento da pesquisa qualitativa: teorias e abordagens. (pp. 15-41). Porto Alegre, BR: Artmed.

Fontanella, B. J. B., Luchesi, B. M., Saidel, M. G. B., Ricas, J., Turato, E. R., \& Melo, D. G. (2011). Amostragem em pesquisas qualitativas: proposta de procedimentos para constatar saturação teórica. Cad. Saúde Pública, 27(2), 388-394. https://doi.org/10.1590/S0102311X2011000200020

Geanellos, R. (2000). Exploring Ricoeur's hermeneu- tic theory of interpretation as a method of analysing research texts. Nursing Inquiry, 7:112-119.

Gadamer, H. G. (2015). Verdade e método (15 $5^{\mathrm{a}}$ ed). Vozes.

Gadamer, H. G. (2006). O caráter oculto da saúde. Petrópolis: Editora Vozes.

Ministério da Saúde. (2021). Banco de dados do Sistema Único de Saúde - SAGE DATASUS. https://sage.saude.gov.br/

Ministério da Saúde. (2021). Banco de dados do Sistema Único de Saúde - TABNET Tipos de estabelecimento. http://tabnet.datasus.gov.br/cgi/cnes/tipo_estabelecimento.htm.

Monteiro, L. D., Mota, R. M. S., Martins-Melo, F. R., Alencar, C. H., \& Heukelbach, J. (2017). Social determinants of leprosy in a hyperendemic State in North Brazil. Rev. de Saúde Pública, 51(4), 51-70. https://doi.org/10.1590/s1518-8787.2017051006655

Patias, N. D., \& Hohendorff, J. V. (2019). Critérios de qualidade para artigos de pesquisa qualitativa. Psicol. Estud, 24(2). http://dx.doi.org/10.4025/psicolestud.v24i0.43536

Ricoeur, P. (2009). Teoria da Interpretação. O discurso e o excesso de significação (1 ${ }^{a}$ ed.). Edições 70 .

Schmitt, J. V., \& Miot, H. A. (2014). Distribution of Brazilian dermatologists according to geographic location, population and HDI of municipalities: an ecological study. Anais Brasileiros de Dermatologia, 89(6), 1013-1015. https://doi.org/10.1590/abd1806-4841.20143276

Silvestre, M. P. S. A., \& Lima, L. N. G. C. (2016). Hanseníase: considerações sobre o desenvolvimento e contribuição (institucional) de instrumento diagnóstico para vigilância epidemiológica. Rev Pan-Amaz Saude, 7(esp Ananindeua), 93-98. 
Vol. 8 | Investigação Qualitativa em Saúde: Avanços e Desafios

Sousa, G. S., Silva, R. L. F., \& Xavier, M. B. (2017). Hanseníase e Atenção Primária à Saúde: uma avaliação de estrutura do programa. Saúde debate, 41(112), 230-242. http://dx.doi.org/10.1590/0103-1104201711219

Starfield, B. (2002). Atenção primária: equilíbrio entre necessidades de saúde, serviços e tecnologia. UNESCO, Ministério da Saúde.

Terra, M. G., Gonçalves, L. H. T., Santos, E. K. A., \& Erdmann, A. L. (2009). Fenomenologiahermenêutica de Paul Ricoeur como referencial metodológico numa pesquisa de ensino em enfermagem. Acta paul. enferm., 22(1), 93-99. https://doi.org/10.1590/S010321002009000100016

Vilela, R., Caldas, L., Correia, B., Almeida, A., Silva, M. A., \& Santos, S. J. (2019). A integralidade do cuidado em saúde na doença falciforme: uso de itinerário terapêutico no apoio à pesquisa qualitativa avaliativa. Atas - Investigação Qualitativa em Saúde, 2(1), 746-755. https://proceedings.ciaiq.org/index.php/CIAIQ2019/article/view/2146/2073

World Health Organization (2016). Global Leprosy Strategy. https://www.who.int/lep/resources/9789290225096/en/ 